04,06

\title{
Ферроэлектрика неоднородно деформированных кристаллов редкоземельных гранатов, возбуждаемая при распространении упругих волн
}

\author{
(С) А.И. Попов ${ }^{1}$, Ч.К. Сабденов ${ }^{2, \uparrow}$, К.А. Звездин ${ }^{2}$ \\ ${ }^{1}$ Национальный исследовательский университет МИЭТ, \\ Москва, Россия \\ ${ }^{2}$ Институт общей ффизики им. А.М. Прохорова РАН, \\ Москва, Россия \\ ฯ E-mail: sabdenovchingiz@gmail.com \\ (Поступила в Редакцию 26 апреля 2017 г.)
}

\begin{abstract}
Проведен анализ влияния деформации на формирование электрических свойств редкоземельных соединений со структурой граната. Показано, что неоднородные деформации приводят к возникновению электрической поляризации у кристаллов гранатов. Эффект обусловлен наличием в структуре кубических кристаллов гранатов неэквивалентных низкосимметричных мест расположения редкоземельных ионов, симметрия окружения которых не содержит центра инверсии. Изучено поведение поляризации подсистемы редкоземельных ионов в кристаллах гранатов при распространении в них упругих волн.
\end{abstract}

Работа поддержана грантом РФФИ № 16-29-14005.

DOI: 10.21883/FTT.2017.11.45070.144

\section{1. Введение}

В настоящее время ведется активный поиск материалов и структур, обладающих магнитоэлектрическими свойствами. Классическими магнитоэлектриками являются мультиферроики (феррит висмута, манганиты, ферробораты, ...), в которых появление магнитоэлектрических свойств обусловлено наличием нечетных конфигураций магнитных моментов $d$-ионов (Fe, $\mathrm{Mn}, \mathrm{Cr}$ ). В последние годы значительно возрос интерес к исследованию редкоземельных мультиферроиков, магнитные свойства которых формируются за счет взаимодействия магнитных подрешеток редкоземельной $(\mathrm{P} 3)(f$-) и железной $(d-)$. Конкуренция между магнитоэлектрическими подсистемами может приводить к нетривиальным физическим эффектам и усилению магнитоэлектрических свойств таких соединений $[9,4]$.

Примером таких материалов, относящихся к новым мультиферроикам, являются Р3 ортоферриты [4]. В данных соединениях Fе-подрешетка является центросимметричной и магнитоэлектрически неактивной, а за возникновение магнитоэлектрических свойств отвечают P3-ионы, магнитные моменты которых могут упорядочиваться в ассиметричные, пространственно-нечетные моды.

К новому классу мультиферроиков с этой точки зрения можно отнести и РЗ-феррит-гранаты (РЗФГ), в которых Р3-подсистема также находится под действием $R$-Feобменного взаимодействия. Большой интерес представляют и парамагнитные гранаты. В работе [6] показано, что в соединении $\mathrm{Mn}_{3} \mathrm{Al}_{2} \mathrm{Si}_{3} \mathrm{O}_{12}$ Р3-подсистема образует антисимметричную антиферромагнитную структуру и в ней может возникнуть электрическая поляризация. В большинстве парамагнитных Р3-гранатов ситуация иная. Они обладают четными АФМ-структурами, и в них магнитное поле индуцирует антисегнетоэлектрические структуры с нулевой электрической поляризацией [6].

Возникновение антисегнетоэлектрической структуры Р3-гранатов обусловлено отсутствием пространственной инверсии окружения Р3-иона, приводящим к индуцированию магнитным полем (порядком) электродипольного момента [6,7].

В настоящей работе показано, что деформация кристалла со структурой граната также приводит к возникновению электрических дипольных моментов у редкоземельных ионов, образующих в случае однородной деформации антисегнетоэлектрическую структуру. Выявлена возможность возникновения электрической поляризации кристаллов гранатов (и их пленок) под воздействием неоднородных механических напряжений. Явление представляет собой неоднородный пьезоэлектрический эффект в центросимметричных средах, содержащих ионы, локальная симметрия окружения которых не содержит пространственной инверсии. Оно может проявляться в пленках редкоземельных гранатов (благодаря неоднородности механических напряжений, образующихся при росте пленок вследствие не полного согласования структур подложки и пленки) и при распространении упругих волн. В настоящей работе проведен анализ электрической поляризации редкоземельных кристаллов гранатов, возникающей при распространении упругих волн. 
Таблица 1. Координаты и оси симметрии додекаэдрических $с$ позиций

\begin{tabular}{c|c|c|c|c|c|c|c|c|c|c|c|c}
\hline $\mathrm{k}$ & 1 & 2 & 3 & 4 & 5 & 6 & 7 & 8 & 9 & 10 & 11 & 12 \\
\hline $\mathbf{r}^{(k)}$ & $0 \frac{3}{4} \frac{3}{8}$ & $0 \frac{1}{4} \frac{1}{8}$ & $\frac{3}{8} 0 \frac{3}{4}$ & $\frac{1}{8} 0 \frac{1}{4}$ & $\frac{3}{4} \frac{3}{8} 0$ & $\frac{1}{4} \frac{1}{8} 0$ & $1 \frac{1}{4} \frac{5}{8}$ & $1 \frac{3}{4} \frac{7}{8}$ & $\frac{5}{8} 1 \frac{1}{4}$ & $\frac{7}{8} 1 \frac{3}{4}$ & $\frac{1}{4} \frac{5}{8} 1$ & $\frac{3}{4} \frac{7}{8} 1$ \\
$\mathbf{e}_{x}^{(k)}$ & 110 & $1 \overline{1} 0$ & 011 & $01 \overline{1}$ & 101 & $\overline{1} 01$ & 110 & $1 \overline{1} 0$ & 011 & $01 \overline{1}$ & 101 & $\overline{1} 01$ \\
$\mathbf{e}_{x}^{(k)}$ & $\overline{1} 10$ & 110 & $0 \overline{1} 1$ & 011 & $10 \overline{1}$ & 101 & $1 \overline{1} 0$ & $\overline{1} \overline{1} 0$ & $01 \overline{1}$ & $0 \overline{1} \overline{1}$ & $\overline{1} 01$ & $\overline{1} 0 \overline{1}$ \\
$\mathbf{e}_{3}^{(k)}$ & 001 & 001 & 100 & 100 & 010 & 010 & 001 & 001 & 100 & 100 & 010 & 010
\end{tabular}

\section{2. Кристаллическая структура Р3-феррит-гранатов}

Редкоземельные (Р3) соединения со структурой граната в течение многих десятилетий являются объектом интенсивных научных исследований, посколько обладают рядом уникальных магнитных, магнитоупругих и магнитооптических свойств, обусловленных в большинстве случаев наличием в их составе РЗ-ионов $[3,8]$. Р3кристаллы со структурой граната имеют общую химическую формулу $\mathrm{R}_{3} \mathrm{M}_{5} \mathrm{O}_{12}$, где $\mathrm{R}$ означает Р3-ион или ион иттрия $\left(\mathrm{Y}^{3+}\right)$. M - ион металла. Ионы металла могут быть двухвалентными $\left(\mathrm{Co}^{2+}, \mathrm{Ga}^{2+}, \mathrm{Mn}^{2+}, \mathrm{Fe}^{2+}\right.$, $\left.\mathrm{Ni}^{2+}\right)$, трехвалентными $\left(\mathrm{Fe}^{3+}, \mathrm{Ga}^{3+}, \mathrm{Al}^{3+}, \mathrm{Se}^{3+}, \mathrm{V}^{3+}\right.$, $\left.\mathrm{Cr}^{3+}\right)$, четырехвалентными $\left(\mathrm{Ge}^{4+}, \mathrm{Si}^{4+}, \mathrm{Zn}^{4+}, \mathrm{Ni}^{4+}, \mathrm{Sn}^{4+}\right)$ и пятивалентными $\left(\mathrm{Nb}^{5+}, \mathrm{P}^{5+}, \mathrm{As}^{5+}\right)[1]$.

Условно гранаты принято разделять на магнитные, у которых $\mathrm{M}$ - ион переходной группы с незаполненной „d“ оболочкой $\left(\mathrm{Fe}^{3+}, \mathrm{Fe}^{2+}, \mathrm{Mn}^{2+}, \mathrm{Co}^{2+}, \mathrm{Cr}^{3+}\right)$, и немагнитные, у которых $\mathrm{M}$ - ионы $\mathrm{Al}^{3+}, \mathrm{Ga}^{3+}, \mathrm{Ge}^{4+}, \mathrm{S}^{4+}$.

Отметим, что структура граната допускает широкие возможности замещения одних ионов (как магнитных, так и немагнитных) на другие. Р3-гранаты являются кубическими магнетиками, обладающими весьма сложной кристаллографической структурой, описываемой пространственной группой $O_{h}^{10}-I a 3 d$. Элементарная ячейка, объемно-центрированная кубическая, включает в себя восемь формульных единиц $\mathrm{R}_{3} \mathrm{M}_{5} \mathrm{O}_{12}$, т.е. 160 атомов. Сторона элементарной ячейки имеет длину $\sim 12 \AA$. Важное значение для нас имеет тот факт, что Р3-ионы в кристаллах гранатов размещены по 6 кристаллографически неэквивалентным местам, обладающим более низкой, чем кубическая симметрией окружения. Симметрия окружения Р3-ионов в гранатах описывается точечной группой $D_{2}$, которая не содержит пространственной инверсии (что является принципиально важным обстоятельством для понимания магнитоэлектрики гранатов $[3,4])$. Для выяснения физических свойств редкоземельных гранатов достаточно ограничиться рассмотрением их примитивной ячейки, которая в два раза меньше элементарной, содержит 4 формульные единицы $\mathrm{R}_{3} \mathrm{M}_{5} \mathrm{O}_{12}$ [8]. Координаты всех 12 Р3-ионов в примитивной ячейке (в единицах ячейки) и ориентации их локальных осей симметрии приведены в табл. 1 (из [6]). Нумерация мест Р3-ионов в ячейке выбрана такой, что окружение места $(k+6)(k=1, \ldots, 6)$ отличается от окружения $k$-го места операцией инверсии, что эквивалентно соотношению $\mathbf{e}_{y}^{(k+6)}=-\mathbf{e}_{y}^{k}$.

Отсутствие пространственной инверсии окружения Р3-иона приводит к индуцированию магнитным полем (порядком) электродипольного момента иона, а, следовательно, и к возникновению структуры электродипольных моментов Р3-ионов [6]. Однако при суммировании по местам, занимаемым РЗ-ионами в примитивной ячейке граната, электрический дипольный момент ячейки в однородном магнитном поле обращается в нуль благодаря операции инверсии, „связывающей“ места $(k+6) \mathrm{c} k$. Отличный от нуля электрический дипольный момент Р-ячейки может возникнуть либо у пленок гранатов, либо в случае неоднородного магнитного поля, действующего на Р3-ионы, на что было указано в [6].

В настоящей работе рассмотрим влияние неоднородных механических напряжений на формирование ферроэлектрических свойств редкоземельных гранатов.

\section{3. Неоднородная деформация и электрическая поляризация редкоземельных ионов}

Представим взаимодействие редкоземельного иона с деформацией, вызванной механическими напряжениями, в виде инвариантного относительно операций симметрии группы $D_{2}$ выражения, представляющего собой произведения компонент тензора деформации $U_{\alpha \beta}$ на компоненты координат $f$-электронов $i$-го иона. В линейном по $\mathbf{r}_{i}$ приближении в локальных осях иона

$$
\begin{aligned}
V_{u} & =q \sum_{i=1}^{N}\left(U_{x y} z_{i}+U_{x z} y_{i}+U_{y z} x_{i}\right) \\
& =q r\left[U_{x y} C_{0}^{1}+\frac{U_{x z}}{\sqrt{2}} i\left(C_{-1}^{1}+C_{1}^{1}\right)+\frac{U_{y z}}{\sqrt{2}} i\left(C_{-1}^{1}-C_{1}^{1}\right)\right],
\end{aligned}
$$

где $q-$ константа взаимодействия (в модели точечных зарядов при учете ионов ближайшего окружения $q=-6 e Q \sum_{k=1}^{8} \frac{R_{k x} R_{k y} R_{k z}}{R^{5}}$, где $Q=2 e-$ заряд иона $O^{2-}, \mathbf{R}_{k}-$ радиусы-векторы ионов $O^{2-}$, окружающих редкоземельный ион), $N$ - число $f$-электронов иона, 
Таблица 2. Компоненты тензора деформации локального окружения редкоземельных ионов в кристаллографической системе координат

\begin{tabular}{c|c|c|c}
\hline$k$ & $U_{x y}^{(k)}$ & $U_{x z}^{(k)}$ & $U_{y z}^{(k)}$ \\
\hline $1 ; 2 ; 7 ; 8$ & $(-1)^{k}\left(U_{X X}-U_{Y Y}\right) / 2$ & $\left(U_{X Z}-(-1)^{k} U_{Y Z}\right) / \sqrt{2}$ & $\left(U_{Y Z}+(-1)^{k} U_{X Z}\right) / \sqrt{2}$ \\
$3 ; 4 ; 9 ; 10$ & $(-1)^{k}\left(U_{Y Y}-U_{Z Z}\right) / 2$ & $\left(U_{X Y}-(-1)^{k} U_{X Z}\right) / \sqrt{2}$ & $\left(U_{X Z}+(-1)^{k} U_{X Y}\right) / \sqrt{2}$ \\
$5 ; 6 ; 11 ; 12$ & $(-1)^{k}\left(U_{Z Z}-U_{X X}\right) / 2$ & $\left(U_{Y Z}-(-1)^{k} U_{X Y}\right) / \sqrt{2}$ & $\left(U_{X Y}+(-1)^{k} U_{Y Z}\right) / \sqrt{2}$
\end{tabular}

$x_{i}, y_{i}, z_{i}$ - координаты $i$-го электрона, $C_{q}^{k}=\sum_{i=1}^{N} C_{q}^{k}(i)$, $C_{q}^{k}(i)$ - одноэлектронный неприводимый тензорный оператор, определяемый приведенным матричным элементом $\left\langle l^{\prime}\left\|C^{p}\right\| l\right\rangle=\sqrt{2 l+1} C_{l 0 p 0}^{l^{\prime} 0}, l^{\prime}$ и $l$ - орбитальные квантовые числа состояний электрона, $C_{a \alpha b \beta}^{c \gamma}-$ коэффициент Клебша-Гордана. Пусть на редкоземельный ион воздействует внешнее электрическое поле Е. Актуальный гамильтониан возмущения запишем в виде

$$
V=-\mathbf{d E}+V_{u},
$$

где $\mathbf{d}=-e \sum_{i=1}^{N} \mathbf{r}_{i}-$ оператор дипольного момента редкоземельного иона.

Линейные по величине $E$ напряженности приложенного электрического поля поправки к уровням энергии иона возникают во втором порядке теории возмущений с соответствующим малым параметром $\|V\| / W$, где $\|V\|$ - норма оператора $V$, а $W-$ разность энергий основных уровней и центра тяжести уровней возбужденных электронных конфигураций иона (для редкоземельных ионов $W \sim 10^{5} \mathrm{~cm}^{-1}$ ), и имеют вид

$$
\begin{aligned}
E_{g}^{(2)}= & \sum_{l^{\prime}, e_{l^{\prime}}} \frac{1}{W_{l^{\prime}}}\left(\left\langle g|\mathbf{d E}| e_{l^{\prime}}\right\rangle\left\langle e_{l^{\prime}}\left|V_{u}\right| g\right\rangle\right. \\
& \left.+\left\langle g\left|V_{u}\right| e_{l^{\prime}}\right\rangle\left\langle e_{l^{\prime}}|\mathbf{d E}| g\right\rangle\right) .
\end{aligned}
$$

Здесь $|g\rangle$ - состояния редкоземельного иона, принадлежащие основной $l^{N}$-конфигурации $(l-$ символ, обозначающий орбитальное квантовое число электронов, для редкоземельного ионов $l=3),\left|e_{l^{\prime}}\right\rangle$ - состояния, принадлежащие возбужденной $l^{N-1} l^{\prime}$-конфигурации с $l^{\prime}=l \pm 1, W_{l^{\prime}}$ - разность энергий состояний $\left|e_{l^{\prime}}\right\rangle$ и $|g\rangle$. При записи (3) мы пренебрегли расщеплением уровней $l^{N-1} l^{\prime}$-конфигурации. К основным уровням ближе расположена конфигурация с $l^{\prime}=l-1=2\left(W_{l-1} \sim 10^{5} \mathrm{~cm}^{-1}\right)$, конфигурация с $l^{\prime}=l+1=4$ лежит выше.

Ограничимся учетом возбужденной конфигурации с $l^{\prime}=l-1$. Используем далее процедуру получения эффективных гамильтонианов, изложенную в [3], и после несложных, но громоздких выкладок найдем, что пьезоэлектрические поправки к уровням энергии (3) могут быть представлены оператором пьезоэлектрического взаимодействия редкоземельного иона (в локальных осях $k$-го места)

$$
\begin{aligned}
& H_{p e}^{(k)}=-A\left(E _ { x } ^ { ( k ) } \left[U_{y z}^{(k)}\left(C_{-2}^{2}+C_{2}^{2}-\sqrt{\frac{2}{3}} C_{0}^{2}\right)\right.\right. \\
& \left.+U_{x z}^{(k)} i\left(C_{-2}^{2}-C_{2}^{2}\right)+U_{x y}^{(k)}\left(C_{-1}^{2}-C_{1}^{2}\right)\right] \\
& +E_{y}^{(k)}\left[U_{y z}^{(k)} i\left(C_{-2}^{2}-C_{2}^{2}\right)-U_{x z}^{(k)}\left(C_{-2}^{2}+C_{2}^{2}+\sqrt{\frac{2}{3}} C_{0}^{2}\right)\right. \\
& \left.+U_{x y}^{(k)} i\left(C_{-1}^{2}+C_{1}^{2}\right)\right]+E_{z}^{(k)}\left[U_{y z}^{(k)}\left(C_{-1}^{2}-C_{1}^{2}\right)\right. \\
& \left.\left.+U_{x z}^{(k)} i\left(C_{-1}^{2}+C_{1}^{2}\right)+U_{x y}^{(k)} 2 \sqrt{\frac{2}{3}} C_{0}^{2}\right]\right)
\end{aligned}
$$

где $A=\frac{e\left(r_{f d}\right)^{2}}{W} \frac{3 \sqrt{3}}{2 \sqrt{70}} q$.

Усредним (4) по состояниям редкоземельных ионов и найдем вклад пьезоэлектрического взаимодействия $k$-го иона в свободную энергию системы

$$
E_{p e}^{(k)}=\left\langle H_{p e}^{(k)}\right\rangle=\frac{\sum_{n}\left\langle\Psi_{n}^{(k)}\left|H_{p e}^{(k)}\right| \Psi_{n}^{(k)}\right\rangle \exp \left(-E_{n}^{(k)} /(k T)\right)}{\sum_{n} \exp \left(-E_{n}^{(k)} /(k T)\right)},
$$

где $E_{n}^{(k)}, \Psi_{n}^{(k)}$ - уровни энергии и волновые функции $k$-го иона. Из (5) легко найти электродипольный момент $k$-го иона (в локальных осях): $P_{\alpha}^{(k)}=-\frac{\partial E_{p e}^{(k)}}{\partial E_{\alpha}^{(k)}}$, $\alpha=x, y, z$. Очевидно, что в случае однородной деформации $P_{\alpha}^{k+6}=-P_{\alpha}^{k}, k=1, \ldots, 6$, так что в данном случае возникают антисегнетоэлектрические конфигурации моментов с нулевым результирующим дипольным моментом.

В кристаллографической системе координат $X=[100]$, $Y=[010], Z=[001]$ компоненты тензора деформации $U_{\alpha \beta}^{(k)}$ имеют вид, представленный в табл. 2.

Средние значения операторов $H_{p e}^{(k)}$ (см. (4), (5)) зависят от состояний ионов в кристалле. Спектр редкоземельных ионов, входящих в состав гранатов, формируется под влиянием кристаллического поля и поля обменного R-Fe-взаимодействия (для редкоземельных ферритгранатов). При этом основным взаимодействием является взаимодействие иона с кристаллическим полем, а обменное R-Fe-взаимодействие (как и взаимодействие с внешним магнитным полем) можно рассматривать в 
качестве возмущения. Исходя их этой иерархии взаимодействий основной вклад в (5) дадут инвариантные относительно операции симметрии группы $D_{2}$ комбинации неприводимых тензорных операторов $C_{-2}^{2}+C_{-2}^{2}$ и $C_{0}^{2}$ в (4), средние от которых отличны от нуля в отсутствие магнитного поля (исключение - ионы $\mathrm{Eu}^{3+}, \mathrm{Gd}^{3+}$ ).

Просуммируем (5) по ионам в ячейке, используя соотношения $U_{\alpha \beta}\left(\mathbf{r}_{k}\right)-U_{\alpha \beta}\left(\mathbf{r}_{k^{\prime}}\right)=\nabla U_{\alpha \beta}\left(\mathbf{r}_{k}-\mathbf{r}_{k^{\prime}}\right)$, и получим пьезоэлектрическую энергию ячейки $E_{p e}$. Далее найдем электрическую поляризацию материала как дипольный момент ячейки, деленный на её объем

$$
P_{\alpha}=-\frac{1}{a^{3}} \frac{\partial E_{p e}}{\partial E_{\alpha}}, \alpha=X, Y, Z,
$$

где $a \sim 12 \AA-$ размер ячейки.

В результате получим

$$
\begin{aligned}
P_{X}= & C_{1}\left(\frac{\partial U_{Y Z}}{\partial Y}+\frac{\partial U_{Y Z}}{\partial Z}-2 \frac{\partial U_{Y Z}}{\partial X}\right) \\
& +C_{2}\left[\left(\frac{1}{2} \frac{\partial U_{X Z}}{\partial Z}+\frac{\partial U_{X Z}}{\partial Y}\right)-\left(\frac{1}{2} \frac{\partial U_{X Y}}{\partial Y}+\frac{\partial U_{X Y}}{\partial X}\right)\right. \\
& \left.+\left(\frac{1}{2} \frac{\partial}{\partial X}+\frac{\partial}{\partial Z}\right)\left(U_{Z Z}-U_{Y Y}\right)\right] \\
P_{Y}= & C_{1}\left(\frac{\partial U_{X Z}}{\partial Z}+\frac{\partial U_{X Z}}{\partial X}-2 \frac{\partial U_{X Z}}{\partial Y}\right) \\
& +C_{2}\left[\left(\frac{1}{2} \frac{\partial U_{X Y}}{\partial X}+\frac{\partial U_{X Y}}{\partial Z}\right)-\left(\frac{1}{2} \frac{\partial U_{Y Z}}{\partial Z}+\frac{\partial U_{Y Z}}{\partial Y}\right)\right. \\
& \left.+\left(\frac{1}{2} \frac{\partial}{\partial Y}+\frac{\partial}{\partial X}\right)\left(U_{X X}-U_{Z Z}\right)\right], \\
P_{Z}= & C_{1}\left(\frac{\partial U_{X Y}}{\partial X}+\frac{\partial U_{X Y}}{\partial Y}-2 \frac{\partial U_{X Y}}{\partial Z}\right) \\
+ & C_{2}\left[\left(\frac{1}{2} \frac{\partial U_{Y Z}}{\partial Y}+\frac{\partial U_{Y Z}}{\partial X}\right)-\left(\frac{1}{2} \frac{\partial U_{X Z}}{\partial X}+\frac{\partial U_{X Z}}{\partial Z}\right)\right. \\
+ & \left.\left(\frac{1}{2} \frac{\partial}{\partial Z}+\frac{\partial}{\partial Y}\right)\left(U_{Y Y}-U_{X X}\right)\right]
\end{aligned}
$$

где

$$
C_{1}=\frac{\left\langle C_{-2}^{2}+C_{2}^{2}\right\rangle A}{a^{2}}, C_{2}=\sqrt{\frac{2}{3}} \frac{\left\langle C_{0}^{2}\right\rangle A}{a^{2}} .
$$

Из (7) следует, что неоднородные деформации кристаллов редкоземельных гранатов могут индуцировать электрическую поляризацию у подсистемы Р3-ионов. В частном случае источником неоднородной деформации являются упругие волны.

\section{4. Упругие волны и волны поляризаций в кристаллах гранатов}

Проведем анализ электрической поляризации, возбуждаемой упругими волнами. Ограничимся рассмотрением объемных волн, распространяющихся вдоль кристаллографических осей симметрии [100], [110], [111]; при этом собственными модами являются одна продольная и две поперечные упругие волны. Рассмотрим также и случай поверхностных волн (волны Рэлея).

Прежде всего отметим, что, как следует из (7), объемные упругие продольные волны, распространяющиеся вдоль высокосимметричных направлений $\langle 100\rangle$ и $\langle 111\rangle$ не приводят к возникновению волн поляризации.

Упругая поперечная волна, распространяющаяся вдоль оси [100] с вектором деформации вдоль направления [001] и амплитудой $U_{0}$ имеет вид $U_{Z}=$ $=U_{0} \cos (k X-\omega t)$. Закон дисперсии для этой волны: $k^{2}(w)=\rho \omega^{2} / c_{44}$, где $\rho-$ плотность, $c_{i k}-$ упругие константы. Искажение описывается компонентой $U_{X Z}(X)$ тензора деформации. В этом случае из (7) получим

$$
\begin{gathered}
P_{X}=0, \\
P_{Y}=\frac{1}{4} C_{1} U_{0} k^{2} \cos (k X-\omega t), \\
P_{Z}=\frac{1}{2} C_{2} U_{0} k^{2} \cos (k X-\omega t) .
\end{gathered}
$$

Для направления [110] рассмотрим последовательно продольную и две поперечные волны. Продольная волна имеет следующие ненулевые компоненты вектора деформации $U_{X}=U_{Y}=\frac{U_{0}}{\sqrt{2}} \cos \left(\frac{k(w)}{\sqrt{2}}(X+Y)-\omega t\right)$, где $k^{2}(w)=\rho \omega^{2} /\left(c_{11}+c_{12}+2 c_{44}\right)$, что приводит к результату

$$
\begin{gathered}
P_{X}=-P_{Y}=C_{2} k^{2}(w) \frac{U_{0}}{\sqrt{2}} \cos \left(\frac{k(w)}{\sqrt{2}}(X+Y)-\omega t\right), \\
P_{Z}=\frac{C_{1}}{2} k^{2}(w) \frac{U_{0}}{\sqrt{2}} \cos \left(\frac{k(w)}{\sqrt{2}}(X+Y)-\omega t\right) .
\end{gathered}
$$

Таким образом, продольная упругая волна при $\mathbf{k} \|\langle 110\rangle$ порождает поперечную волну поляризации. Для поперечной упругой волны, „лежащей“ в плоскости $O X Y$, у которой $U_{X}=-U_{Y}=\frac{U_{0}}{\sqrt{2}} \times$

$$
\begin{gathered}
\times \cos \left(i\left(\frac{k(w)}{\sqrt{2}}(X+Y)-\omega t\right)\right) \\
P_{X}=P_{Y}=C_{2} k^{2}(w) \frac{U_{0}}{2 \sqrt{2}} \cos \left(\frac{k(w)}{\sqrt{2}}(X+Y)-\omega t\right), \\
P_{Z}=0, k^{2}(w)=\rho \omega^{2} /\left(c_{11}-c_{12}\right) .
\end{gathered}
$$

В случае поперечной волны, вектор деформации которой перпендикулярен плоскости $O X Y, U_{Z}=U_{0} \times$ $\times \cos \left(\frac{k(w)}{\sqrt{2}}(X+Y)-\omega t\right)$

$$
\begin{gathered}
P_{X}=P_{Y}=-\frac{1}{8} C_{1} k^{2}(w) U_{0} \cos \left(\frac{k(w)}{\sqrt{2}}(X+Y)-\omega t\right) \\
P_{Z}=0, \quad k^{2}(w)=\rho \omega^{2} / c_{44}
\end{gathered}
$$

Из (10) и (11) следует, что поперечные упругие волны, распространяющиеся в направлениях $\langle 110\rangle$, порождают продольные волны поляризации. 
При $\mathbf{k}$ || [111] поперечная упругая волна, для которой $U_{X}=-U_{Y}=\frac{U_{0}}{\sqrt{2}} \cos \left(\frac{k(w)}{\sqrt{3}}(X+Y+Z)-\omega t\right), U_{Z}=0$, где $k^{2}(w)=\rho \omega^{2} /\left(c_{11}-c_{12}+c_{44}\right), \quad$ возбуждает поляризацию

$$
\begin{aligned}
P_{X} & =P_{Y}=-P_{Z} / 2 \\
& =-C_{2} k^{2}(w) \frac{U_{0}}{2 \sqrt{2}} \cos \left(\frac{k(w)}{\sqrt{3}}(X+Y+Z)-\omega t\right) .
\end{aligned}
$$

И, наконец, поперечная волна с вектором деформации, направленным вдоль $[11 \overline{2}]$, возбуждает поляризацию

$$
\begin{aligned}
P_{X} & =-P_{Y}=\frac{3}{2 \sqrt{6}} C_{2} \\
& \times \cos \left(\frac{k(w)}{\sqrt{3}}(X+Y+Z)-\omega t\right) k^{2}(\omega) U_{0}, P_{Z}=0 .
\end{aligned}
$$

Данное направление характерно тем, что три вектоpa $\mathbf{P}, \mathbf{k}, \mathbf{U}$ перпендикулярны друг другу.

Перейдем теперь к рассмотрению поверхностных упругих волн. Пусть поверхность лежит в плоскости (001), а в направлении [100] распространяется поверхностная волна $\mathbf{U}=\mathbf{U}_{l}+\mathbf{U}_{t}$, являющаяся суперпозицией продольной $\left(\mathbf{U}_{l}\right)$ и поперечной $\left(\mathbf{U}_{t}\right)$ компонент, связанных следующими соотношениями (см., например, [9])

$$
\begin{gathered}
U_{t x}=\chi_{t} a \cos (k x-\omega t) \exp \left(\chi_{t} z\right), \\
U_{t z}=k a \sin (k x-\omega t) \exp \left(\chi_{t} z\right), \\
U_{l x}=k b \cos (k x-\omega t) \exp \left(\chi_{l} z\right), \\
U_{l z}=\chi_{l} b \sin (k x-\omega t) \exp \left(\chi_{l} z\right), \\
\chi_{l, t}=\left(k^{2}-\frac{\omega^{2}}{c_{l, t}^{2}}\right)^{\frac{1}{2}}, \\
2 a \chi_{t} k+b\left(k^{2}+\chi_{t}^{2}\right)=0,\left(k^{2}+\chi_{t}^{2}\right)^{2}=4 k^{2} \chi_{t} \chi_{l} .
\end{gathered}
$$

Воспользуемся формулой (7) и вычислим отдельно компоненты вектора поляризации, произведенные поперечной и продольной составляющими. В результате получим

$$
\begin{gathered}
P_{X}^{(t)}=\frac{1}{2} a C_{2} \chi_{t}\left(3 k^{2}+\chi_{t}^{2}\right) \cos (k x-\omega t) \exp \left(\chi_{t} z\right), \\
P_{Y}^{(t)}=-\frac{1}{4} a C_{1}\left(k^{2}+\chi_{t}^{2}\right)\left(\chi_{t} \cos (k x-\omega t)\right. \\
-k \sin (k x-\omega t)) \exp \left(\chi_{t} z\right), \\
P_{Z}^{(t)}=\frac{1}{2} a C_{2} k\left(k^{2}+3 \chi_{t}^{2}\right) \sin (k x-\omega t) \exp \left(\chi_{t} z\right), \\
P_{X}^{(l)}=2 b C_{2} k \chi_{l}^{2} \cos (k x-\omega t) \exp \left(\chi_{l} z\right), \\
P_{Y}^{(l)}=-\frac{1}{2} b C_{1} k \chi_{l}\left(\chi_{l} \cos (k x-\omega t)\right. \\
-k \sin (k x-\omega t)) \exp \left(\chi_{l} z\right), \\
P_{Z}^{(l)}=2 b C_{2} k^{2} \chi_{l} \sin (k x-\omega t) \exp \left(\chi_{l} z\right) .
\end{gathered}
$$

\section{5. Заключение}

Таким образом, в работе показано, что деформации редкоземельных кристаллов со структурой граната вызывают появление электрических дипольных моментов у редкоземельных ионов. Данное обстоятельство обусловлено наличием в структуре кубических кристаллов гранатов неэквивалентных мест для редкоземельных ионов, симметрия окружения которых не содержит центра инверсии. В случае однородных деформаций дипольные моменты редкоземельных ионов образуют антисегнетоэлектрические структуры с нулевым результирующим электрическим дипольным моментом ячейки. В то время, как неоднородные деформации в общем случае служат источником возникновения электрической поляризации. Явление представляет собой неоднородный пьезоэффект. Простейшей возможностью реализации неоднородных деформаций являются упругие волны. В настоящей работе исследована электрическая поляризация редкоземельных кристаллов гранатов, возникающая при распространении в них упругих волн. Установлено, что продольные упругие волны, распространяющиеся в объеме в высокосимметричных направлениях $\langle 100\rangle$ и $\langle 111\rangle$ не приводят к возникновению поляризации, в то время как продольные волны, распространяющиеся вдоль осей $\langle 110\rangle$ индуцируют поперечные волны поляризации. Поперечные упругие волны в случае $\mathbf{k} \|\langle 110\rangle$ порождают продольные волны поляризации. При $\mathbf{k} \|\langle 100\rangle$ и $\mathbf{k} \|\langle 111\rangle$ поперечные упругие волны в объеме возбуждают поперечные волны поляризации, причем для $\mathbf{k} \|\langle 111\rangle$ все три вектора $\mathbf{k}, \mathbf{U}, \mathbf{P}$ взаимно перпендикулярны. В случае поверхностных волн подобные простые закономерности отсутствуют.

\section{Список литературы}

[1] Y. Tokunaga, Y. Taguchi, T.-h. Arima, Y. Tokura. Nature Phys. 8, 1, 838 (2012).

[2] А.К. Звездин, А.А. Мухин. Письма в ЖЭТФ 88, 8, 505 (2008).

[3] A.I. Popov, D.I. Plokhov, A.K. Zvezdin. Phys. Rev. B 90, 21, 4427 (2014).

[4] A.I. Popov, Z.V. Gareeva, A.K. Zvezdin. Phys. Rev. B 92, 14, 4420 (2015).

[5] А.К. Звездин, В.М. Матвеев, А.А. Мухин, А.И. Попов. Редкоземельные ионы в магнитоупорядоченных кристаллах. (1985). 296 c.

[6] A.K. Zvezdin, V.A. Kotov. Modern magnetooptics and magnetooptical materials. CRC Press (1997). $404 \mathrm{c}$.

[7] К.П. Белов, Н.А. Белянчикова, Р.3. Левитин, С.А. Никитин. Редкоземельные феррои антиферромагнетики. Наука, М. (1965). $449 \mathrm{c}$.

[8] Ю.А. Изюмов, Р.П. Озеров, В.Е. Найш. Нейтронография магнетиков. Атомиздат, М. (1981). 311 с.

[9] Л.Д. Ландау, Е.М. Лифшиц. Теоретическая физика. Теория упругости. Наука, М. (1989). Т. 7. 264 с. 